\title{
Concurrent sequential matching in the pigeon
}

\author{
RICHARD PISACRETA \\ Ferris State College, Big Rapids, Michigan 49307
}

\begin{abstract}
Two pigeons were trained to peck two pairs of matching stimuli in sequence. Each trial presented four illuminated keys, two pairs of matching stimuli. A response to any key darkened the key. A response to the other key presenting the same stimulus that was extinguished by the first response darkened that key. A response to each of the remaining two keys presenting the other matching stimuli darkened those keys and produced a fifth key illuminated with white. A response to this key produced 3 sec access to grain, a 3-sec intertrial interval (ITI), and the next trial. An error (i.e., pecking two different stimuli) produced a 5-sec time-out (TO). During Phase 1, two of three colors (red, green, and yellow) were presented on each trial. Phase 2 used combinations of two forms and a color: an "X," a circle, and yellow. Phase 3 employed three novel stimuli: horizontal line, vertical line, and blue. Both birds showed an initial increase in errors across phases. Both pigeons demonstrated first-response stimulus preferences (i.e., responded to green first during Phase 1, yellow during Phase 2, and blue during Phase 3). The various spatial configurations of keys and stimuli influenced the errors made by both birds.
\end{abstract}

One index for the assessment of animal intelligence is a species' ability to learn complex temporal and spatial associations between stimuli and responses. For this reason, among others, the matching-to-sample procedure has been frequently explored (Cumming \& Berryman, 1965; D'Amato, 1973; Honig, 1978). Farthing and Opuda (1974) provided three possibilities concerning what is learned during the acquisition of matching to sample. (1) Pigeons learn a matching concept. Conceptual behavior involves generalization within classes of stimuli and discrimination between stimulus classes (Keller \& Schoenfeld, 1950, p. 155). This position implies that pigeons should maintain accurate matching when exposed to novel stimuli. (2) Pigeons learn a set of chains, for example, "Peck blue on the center key, then peck blue on a side key." This position predicts that each novel standard stimulus introduced into the matching situation will produce errors until the new chain is acquired. Pigeons should match well, however, with novel comparison stimuli because the novel stimulus is not part of the original matching chain. (3) Pigeons learn sets of spatial discriminations, for example, "Given the horizontal key arrangement green-red-red, peck the center and right keys." This position predicts that pigeons must learn appropriate behavior in each novel stimulus situation as a different discrimination.

Several lines of research have provided support for each of these positions (e.g., Carter \& Eckerman, 1975; Cumming, Berryman, \& Cohen, 1965; D'Amato, 1973; Farthing \& Opuda, 1974; Urcuioli \& Nevin, 1975). Several studies have manipulated various aspects of the

I thank Coleman Paul for his support and contributions to this work and Nancy Myers for typing various drafts. Reprints may be obtained from Richard Pisacreta, Department of Psychology, Ferris State College, Big Rapids, Michigan 49307. matching paradigm in attempts to improve acquisition or transfer of matching while providing support for one of the three positions. Manipulations have included sample stimulus duration (e.g., Devine, Jones, Neville, \& Sakai, 1977; Grant, 1976) and compound sample stimuli (Make \& Leith, 1973). Urcuioli and Nevin (1975) proposed that inability to transfer matching may be due to the birds' failure to learn which comparison stimuli are incorrect or nonmatching. With their procedure, only one of the comparison stimuli was presented after a response was made to the sample stimulus. If the stimulus matched the standard stimulus and the bird pecked it, a reinforcer was delivered. If the nonmatching stimulus occurred first, it was replaced by the matching stimulus if the bird did not respond to it within $4.8 \mathrm{sec}$. Responses to nonmatching stimuli reset the 4.8-sec interval. When novel samples were introduced, the birds demonstrated transfer of matching (i.e., the concept of hue matching).

The present study presented pigeons with four stimuli in two matching pairs. Each trial presented these stimuli in various spatial configurations by employing combinations of six response keys. Reinforcement was contingent upon the birds' pecking the stimuli in a matching sequence. The procedures used attempts to teach pigeons to discriminate nonmatching stimulusresponse combinations by delivering a time-out (darkened chamber) followed by a resetting of the same trial for incorrect responses. The procedure allows birds to choose the order of matching by providing two sets of sample stimuli and therefore enables the birds to demonstrate matching preferences. After the first response, the trial resembles matching to sample with two nonmatching and one matching stimulus. Specifically, the present study attempted to establish a "conditional" discrimination in which the appropriate response is defined by 
two or more features of the environment, which, in turn, are dictated by the pigeon's ongoing behavior. Various novel stimuli were later introduced to assess the three interpretations of the pigeons' matching to sample in this context.

\section{METHOD}

Subjects

Two naive White Carneaux pigeons were maintained at $80 \% \pm 15 \mathrm{~g}$ of their free-feeding weight.

\section{Apparatus}

The apparatus was a $35 \times 35 \times 37 \mathrm{~cm}$ operant chamber enclosed in a sound-attenuating hull. A $20 \times 25 \mathrm{~cm}$ piece of oneway glass mounted on the door of the outer hull enabled observation of the entire experimental area.

The nine-response-key intelligence panel, $37 \times 35 \mathrm{~cm}$, employed standard dimensions and commercial components. The keys were arranged in a 3 by 3 matrix. For reference, the keys are numbered from upper left (1) across to lower right (9). Each key was $2.7 \mathrm{~cm}$ in diameter (BRS/LVE Model 121-16). Stimuli were projected onto the keys by Industrial Electronics Engineers in-line projectors (Model 1813-44). The operatingforce requirement of each was approximately $.16 \mathrm{~N}$. The horizontal and vertical distances between the keys were 8.1 and $6.4 \mathrm{~cm}$, respectively, center to center. A $6 \times 6 \mathrm{~cm}$ feeder aperture was centered on the wall $10 \mathrm{~cm}$ above the floor. The feeder (BRS/LVE Model 114-10) provided $3 \mathrm{sec}$ access to grain. The houselight, a GE 1820 lamp, provided light before and after daily experimental sessions. During sessions, illumination was provided only by the in-line projectors. Response Keys 1 and 3 were never illuminated. White noise delivered through a speaker and a ventilation fan masked extraneous noise. An $E$ and $L$ Instruments MMD-1 computer recorded data and controlled experimental events.

\section{Procedure}

Preliminary training. Both subjects were magazine trained. Pecking Key 2, which presented a white field as the discriminative stimulus (SD), was shaped. Each bird was allowed to produce 100 food presentations under these conditions. Sessions for the remainder of the experiment terminated after 45 reinforcements. The birds were then trained to peck pairs of stimuli. The lower six response keys $(4,5,6,7,8$, and 9) were employed. On each trial, two of the six keys were illuminated with yellow. A response to either key darkened that key. When each yellow key was extinguished, Key 2 was illuminated with white. A response to this key produced a reinforcer, a 3-sec intertrial interval (ITI), and the next trial. During the ITI, all chamber illumination was eliminated. The four sessions presenting yellow pairs were followed by four sessions of green pairs and then four sessions of red pairs of stimuli.

Matching pairs of stimuli. For the next 30 sessions (Phase 1), the pigeons were trained to peck pairs of matching stimuli. Each trial presented four illuminated keys. The keys presented two pairs of matching colors. For example, on some trials, Key 4 was red, 5 was green, 6 was green, and 7 was red. A response to any key darkened that key. A response to the other key presenting the same color that was extinguished by the first response darkened that key as well. A response to each of the remaining two keys, presenting the other color match, darkened those keys and produced white on Key 2. As before, a response to Key 2 produced a reinforcer, an ITI, and the next trial. An incorrect response (pecking two different stimuli) produced a 5 -sec time-out followed by a recycling of the trial (i.e., reillumination of the four keys). All chamber illumination was eliminated during the time-out. During this phase, the color pairs were 15 randomized trials each of red-green, red-yellow, and green-yellow.

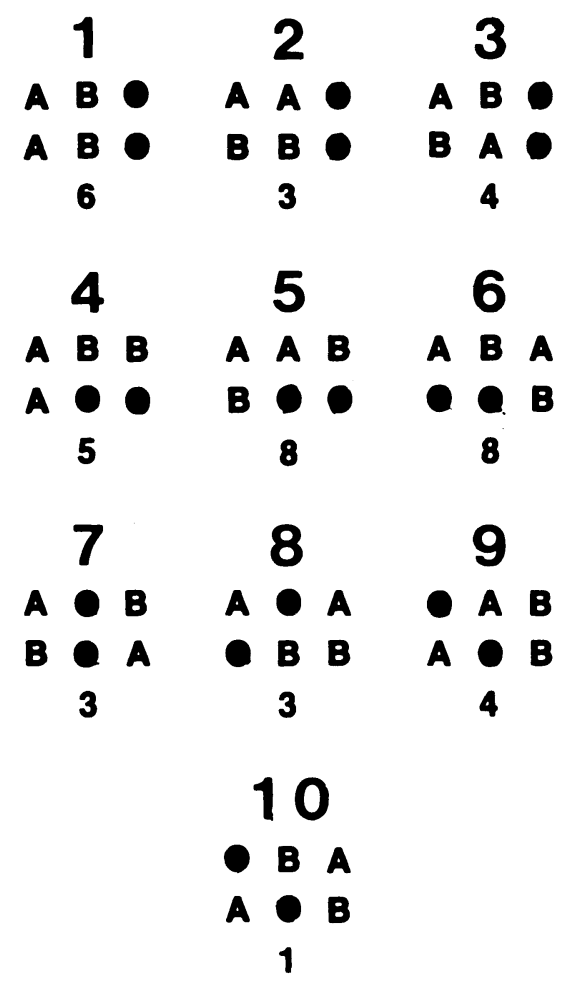

Figure 1. The 10 response-key configurations presented on Keys 4-9 inclusive. " $A$ " refers to one stimulus pair; " $B$ " represents the other, matching pair of stimuli. Filled circles represent darkened keys. The large number above each configuration labels the pattern for reference. The smaller number below each pattern indicates the number of trials per session that the configuration was used.

Figure 1 shows the spatial configurations employed in all three phases of the experiment. " $A$ " and " $B$ " represent the two matching pairs of stimuli. For example, a red-green trial using Pattern 5 presented red on Keys 4 and 5 (A) and green on Keys 6 and 7 (B). A green-yellow trial using Pattern 6 offered green on Keys 4 and 6 and yellow on Keys 5 and 9.

After 30 sessions, the red and green stimuli were replaced by two forms, a white $\mathrm{X}$ and a white circle $(\mathrm{O})$ on dark backgrounds. The stimulus pairs used during the next 20 sessions (Phase 2) were X-O, X-yellow, and O-yellow. This phase utilized a familiar stimulus (yellow) with two novel forms.

Phase 3 employed three novel stimuli for the remaining 20 sessions of the experiment. They were a blue field, a white vertical $(V)$ line, or a white horizontal $(H)$ line on a black background. Trials consisted of the stimulus pairs V-H, V-blue, and H-blue.

\section{RESULTS}

Figure 2 shows errors as a function of stimulus pairs. Both birds produced similar error patterns. During Phase 1 , both birds made more errors with red-yellow and green-yellow trials than with red-green trials. During Phase 2, more errors were produced during the trials presenting two forms than during trials employing colorform combinations. This result held during Phase 3 . There were fewer errors on trials with color-form matching pairs. 


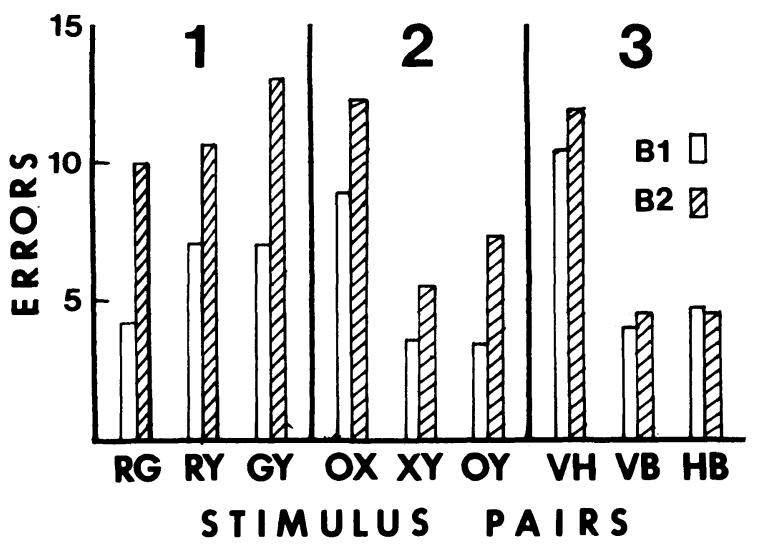

Figure 2. Errors (hundreds) produced as a function of stimulus pairs. The stimulus pairs include: red-green (RG), redyellow (RY), green-yellow (GY), circle-X (OX), X-yellow (XY), circle-yellow (OY), vertical line/horizontal line (VH), vertical line/blue (VB), and horizontal line/blue (HB).

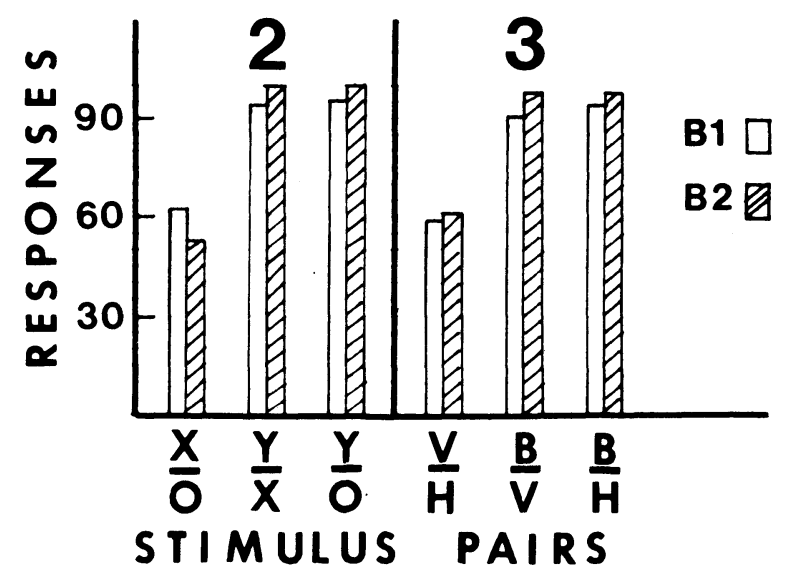

Figure 3. Stimulus preference during Phases 2 and 3. The bars represent the number of times (of 105 trials) that the birds responded first to the upper stimulus on the abscissa. The lower stimulus was the alternate choice. From left to right, they are: $X$-circle, yellow-X, yellow-circle, vertical line/horizontal line, blue/vertical line, and blue/horizontal line.

Which stimulus was pecked first on a trial was analyzed for the first session of each phase and every third session thereafter (Sessions 4, 7, 10, etc.). During Phase 1, both birds pecked green first on every trial presenting red-green or yellow-green. When presented with redyellow, the birds pecked red and yellow first equally often. Figure 3 depicts the data for the other two phases. The data represent seven sessions of each phase $(1,4,7,10,13,16$, and 19). Since each stimulus pair was used for 15 trials/session, the seven sessions represent 105 opportunities to demonstrate a preference for particular matches. The bars show the cumulative number of times that a bird chose the upper stimulus of each pair. Both birds almost exclusively pecked a color first when confronted with color-form pairs on a trial. During the 105 trials of each type in Phase 2, B1 chose yellow over X 94 times and yellow over circle 96 times.
B2 chose yellow over X 99 times and yellow over circle 100 times. Similarly, in Phase 3, B1 pecked blue first 91 and 94 times instead of the vertical line or horizontal line, respectively. B2 chose blue 96 and 98 times before responding to the form stimuli. When two forms were presented, the birds yielded no consistent preference. A comparison of Figures 2 and 3 reveals that both birds made fewer errors on trials in which they showed a consistent stimulus preference. These parsimonious response patterns demonstrated that a reinforcer influences not only the response that produces it (the fifth response to white on Key 2), but also the four responses that were never directly followed by reinforcement (Catania, 1971; Hawkes \& Shimp, 1975; Pisacreta, 1982; Schwartz, 1980; Wasserman, 1977). Specifically, strict response-reinforcer contiguity may not be essential for conditioning to occur (Shimp, 1976a, 1976b; Wasserman, 1977).

Finally, Figure 4 displays the distributions of errors as a function of the types of spatial configurations used. The uneven distributions of errors indicates that the spatial arrangement of the stimuli influenced the number of errors. As in Figures 2 and 3, the data from both birds are similar. During Phase 1 , both subjects made the greatest number of errors with Trial Types 5 and 6 . These trials required the birds to "pass over" some keys
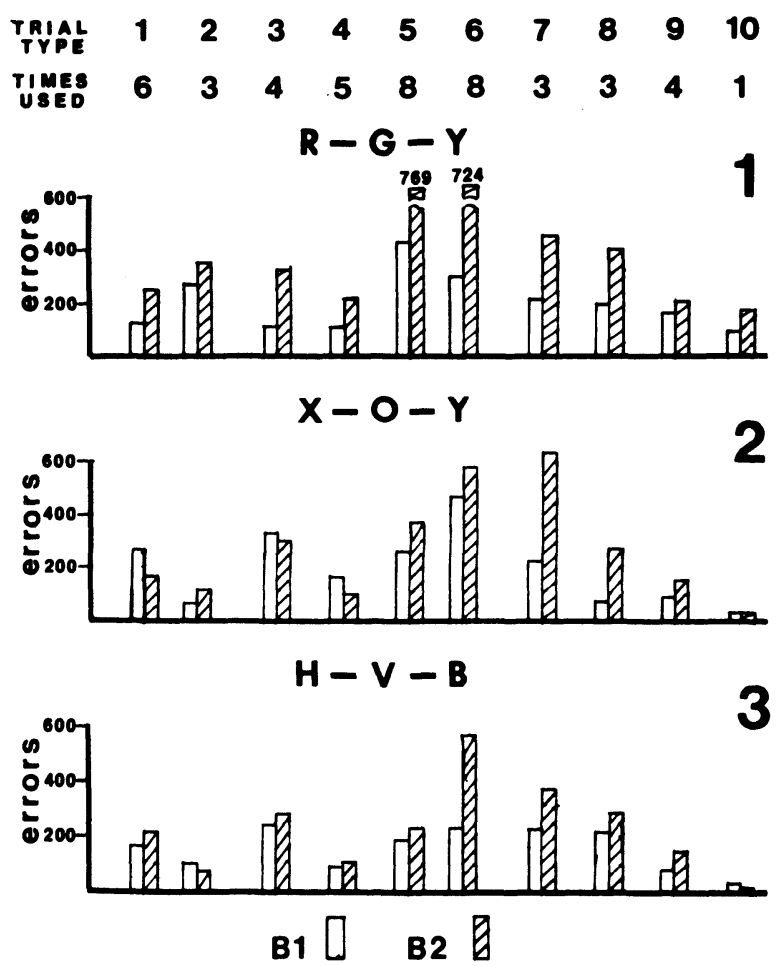

Figure 4. Errors as a function of spatial configurations used. The upper panel presents Phase 1, red-green-yellow stimuli. The center panel shows Phase 2, X-circle-yellow stimuli and the lower panel displays the horizontal line/vertical line/blue stimuli of Phase 3. The trial-type numbers correspond to those displayed in Figure 1. "Times used" refers to the number of times each configuration occurred during each 45-trial session. 
in order to peck the stimulus that matched the stimulus they had just pecked. Stimulus arrangements that displayed the matching stimuli vertically (Types 1 and 9), horizontally (Types 2 and 8), and diagonally (Types 3, 7 , and 10) yielded comparatively fewer errors. Changing the matching stimuli in Phase 2 produced changes in the distributions of errors. During this phase, B1 produced more errors on Trial Types 1, 3, 4, and 6 and a decrease in errors for Trial Types 2, 8, 9, and 10. B2 showed a decrease in errors for all trial types except 3 and 7 . These results may reflect the emergence of the matching preferences depicted in Figure 3 . Phase 3 produced slight changes in error distributions. B1 made more errors on Trial Types 2 and 8 and fewer on Trial Types 1, 3, 4, and 6. B2 made fewer errors on Trial Types 2, 5, and 7 and more on Trial Type 1.

\section{DISCUSSION}

The present procedure simultaneously employed matching to sample and oddity. After the first response is emitted, the bird is left with a matching pair of stimuli and a nonmatching stimulus that is the correct stimulus to peck. Considering the number of keys and stimuli involved, the birds showed a large degree of response stereotypy. Although variability was permitted (i.e., there were eight different response sequences that will produce a reinforcer on any trial), each bird quickly showed a dominant sequence for each trial type. Schwartz (1981) provided data indicating that self-determined systematic sequences are primarily due to visual feedback for specific responses. Schwartz suggested that "response sequences that involve more motorically heterogeneous components may be more easily controlled by proprioceptive information" (1981, p. 43). The present study, which required the birds to shuttle across six keys to peck four keys lit in various spatial configurations, appears to support this view.

Skinner (1950) proposed that matching to sample involves the learning of simple associative rules. Matching to sample with red and green stimuli is learned by acquiring two discriminative responses: "peck red after being stimulated by red." The matching preferences that emerged in the present study lend support to this view. The initial increase in errors when novel stimuli were introduced would seem also to support the discrimination position, instead of the "concept learning" and "concommitant chains" positions cited in the introduction. The discriminative response position has received support from several studies (e.g., Carter \& Werner, 1978; Cumming \& Berryman, 1961; Farthing \& Opuda, 1974). It is tempting, however, to speculate that the birds were emitting the chain "peck the colors and then the forms" during Phases 2 and 3. The change from "peck green first" (Phase 1) to "peck yellow first" (Phase 2) to "peck blue first" (Phase 3) implies that the rule "peck a particular color" was in evidence.

Several researchers have provided data to show that functional operants may include responses arranged into temporal and spatial groups, that is, that several of the responses that precede a reinforcer may be affected by the reinforcer (e.g., Grayson \& Wasserman, 1979; Hawkes \& Shimp, 1975; Pisacreta, 1982; Wasserman, 1977). The present results are consistent with Shimp's (1976) position: "The repeated delivery of a reinforcer after a behavioral pattern a few seconds in duration may strengthen the entire pattern and establish it as a functional unit. In other words, a pigeon learns one or more patterns of key pecks, not just a key peck" (p. 60). Furthermore, the results of the present study may be interpreted as support for the position that the tenets of operant conditioning are applicable to spatially complex, organized sequences of behavior.

\section{REFERENCES}

Carter, D. E., \& Eckerman, D. A. Symbolic matching by pigeons: Rate of learning complex discrimination predicted from simple discriminations. Science, 1975, 187, 662-664.

Carter, D. E., \& Werner, T. J. Complex learning and information processing. A critical analysis. Journal of the Experimental Analysis of Behavior, 1978, 29, 656-701.

Catania, A. C. Reinforcement schedules: The role of responses preceding the one that produces the reinforcer. Journal of the Experimental Analysis of Behavior, 1971, 15, 271-288.

Cumming, W. W., \& Berryman, R. Some data on matching behavior in the pigeon. Journal of the Experimental Analysis of Behavior, 1961, 4, 281-284.

Cumming, W. W., \& Berryman, R. The complex discriminated operant: Studies of matching-to-sample and related problems. In D. E. Mostofsky (Ed.), Stimulus generalization. Stanford, Calif: Stanford University Press, 1965.

Cumming, W. W., Berryman, R., \& Cohen, L. R. Acquisition and transfer of zero-delay matching. Psychological Reports, $1965,17,435-445$.

D'Aмлто, M. R. Delayed matching and short term memory in monkeys. In G. H. Bower (Ed.), The psychology of learning and motivation: Advances in research and theory (Vol. 7). New York: Academic Press, 1973.

Devine, J. V., Jones, L. C., Neville, J. W., \& Sakai, D. J. Sample duration and type of stimuli in delayed matching to sample in rhesus monkeys. Animal Learning \& Behavior, 1977, 5, 57-62.

Farthing, G. W., \& Opuda, M. J. Transfer of matching to sample in pigeons. Journal of the Experimental Analysis of Behavior, 1974, 21, 199-213.

Grant, D. S. Effect of sample presentation time on long delay matching in the pigeon. Learning and Motivation, 1976, 7 , 580-590.

Grayson, R. J., \& Wasserman, E. A. Conditioning of tworesponse patterns of key pecking in pigeons. Journal of the Experimental Analysis of Behavior, 1979, 31, 23-29.

HAwkes, L., \& SHIMP, C. P. Reinforcement of behavioral patterns: Shaping a scallop. Journal of the Experimental Analysis of Behavior, 1975, 23, 3-16.

Honig, W. K. Studies of working memory in the pigeon. In S. H. Hulse, H. Fowler, \& W. H. Honig (Eds.), Cognitive processes in animal behavior. Hillsdale, N.J: Erlbaum, 1978.

Keller, F. S., \& Schoenfeld, W. N. Principles of psychology. New York: Appleton-Century-Crofts, 1950.

MAKE, W. S., \& LEITH, C. R. Shared attention in pigeons. Journal of the Experimental Analysis of Behavior, 1973, 19, 345-349.

Pisacreta, R. Some factors that influence the acquisition of complex, stereotyped responses in pigeons. Journal of the Experimental Analysis of Behavior, 1982, 37, 359-369.

Schwartz, B. Development of complex, stereotyped behavior in pigeons. Journal of the Experimental Analysis of Behavior, $1980,33,153-166$.

Schwartz, B. Control of complex, sequential operants by systematic visual information in pigeons. Journal of Experimental Psychology: Animal Behavior Processes, 1981, 1, 31-44.

Shimp, C. P. Organization in memory and behavior. Journal of the Experimental Analysis of Behavior, 1976, 26, 113-130. (a)

SHIMP, C. P. Short term memory in the pigeon: Relative recency. Journal of the Experimental Analysis of Behavior, 1975, 25, 55-61. (b)

Skinner, B. F. Are theories of learning necessary? Psychological Review, 1950, 57, 193-206.

Urcuioli, P. J., \& Nevin, J. A. Transfer of hue matching in pigeons. Journal of the Experimental Analysis of Behavior, 1975, 24, 149-155.

Wasserman, E. A. Conditioning of within trial patterns of key pecking in pigeons. Journal of the Experimental Analysis of Behavior, 1977, 28, 213-220.

(Received for publication July 14, 1982.) 